\title{
Conhecimento, atitude e prática sobre fototerapia entre profissionais de Enfermagem de hospitais de ensino
}

\author{
Knowledge, attitude and practice about phototherapy among nursing professionals of teaching hospitals
} Conocimiento, actitud y práctica en fototerapia entre profesionales de enfermería de hospitales de enseñanza

\author{
Simone Pires Cavalcanti Machado', Isabella Chagas Samico", Taciana Duque de Almeida Braga"I' \\ IInstituto de Medicina Integral Prof. Fernando Figueira, Unidade Neonatal. Recife-PE, Brasil. \\ "Instituto de Medicina Integral Prof. Fernando Figueira, Programa de Pós-Graduação em Avaliação em Saúde. Recife-PE, Brasil. \\ II' Faculdade Pernambucana de Saúde, Curso de Medicina. Recife-PE, Brasil.
}

\section{Submissão 12-08-2010 Aprovação: 01-03-2012}

\section{RESUMO}

Este artigo consiste em um estudo tipo inquérito CAP (Conhecimento, Atitude e Prática) com objetivo de avaliar o conhecimento, atitude e prática sobre fototerapia entre profissionais de Enfermagem de dois hospitais de ensino da cidade de Recife-PE, por meio de questionário semiestruturado aplicado a 111 profissionais. Para comparação das médias de acertos, foi utilizado o teste $t$ de Student. Assim, verificaram-se maiores médias para a prática, seguidas das relacionadas à atitude e menores percentuais de acertos para o conhecimento. Na comparação das médias entre conhecimento e prática, as diferenças se mostraram estatisticamente significantes para todas as variáveis $(p<0,05)$. Os resultados do estudo aqui apresentado apontam para a necessidade de implementação de atividades capazes de permitir um equilíbrio entre conhecimento, atitude e prática dos profissionais de Enfermagem sobre fototerapia.

Descritores: Conhecimento, atitude e prática em saúde; Pesquisa em Enfermagem; Icterícia; Fototerapia; Recém-Nascido.

\begin{abstract}
This article consists of a KAP (Knowledge, Attitude and Practice) survey to evaluate knowledge, attitude and practice of phototherapy among nursing professionals from two teaching hospitals at the city of Recife, Brazil, through semi-structured questionnaire applied to 111 professionals. It was used the T Student test to compare means of correct answers. Thus, there were higher means for practice, followed by those related to attitude and a lower percentage of correct answers for knowledge. When comparing the means between knowledge and practice the differences were statistically significant for all variables $(p<0.05)$. The results of the study highlight the need to implement activities that will allow a balance among knowledge, attitude and practice of nursing professionals about phototherapy.
\end{abstract}

Key words: Health knowledge, attitudes and practice; Nursing Research; Jaundice; Phototherapy; Newborn.

\section{RESUMEN}

Este artículo consiste en un estudio de tipo investigación CAP (conocimiento, actitud y práctica) con el objetivo de evaluar lo conocimiento, actitude y práctica sobre fototerapia entre profesionales de enfermería de dos hospitales de enseñanza de la ciudad de Recife, Brasil, mediante cuestionario semi-estructurado a 111 profesionales. Para la comparación de la media de respuestas correctas, se utilizó la prueba $t$ de Student. Por lo tanto, se registraron mayores medios para la práctica, seguidos por los relacionados con la actitud y el menor porcentaje de respuestas correctas para el conocimiento. Al comparar las medias entre el conocimiento y la práctica, las diferencias fueron estadísticamente significativas para todas las variables $(p<0,05)$. Los resultados del estudio apuntan a la necesidad de implementar actividades que permitan un equilibrio entre el conocimiento, actitude y práctica de los profesionales de enfermería acerca de la fototerapia.

Palabras clave: Conocimiento, actitud y práctica en salud; Investigación en enfermería; Ictericia; Fototerapia; Recién Nacido. 


\section{INTRODUÇÃO}

A hiperbilirrubinemia indireta é um dos problemas mais comuns encontrados no período neonatal ${ }^{(1)}$. Estima-se que cerca de $60 \%$ dos recém-nascidos desenvolvem níveis séricos de bilirrubina acima de $5 \mathrm{mg} \%$ e aproximadamente 5 a $10 \%$ têm hiperbilirrubinemia clínica significante ${ }^{(2)}$. Apesar da hiperbilirrubinemia neonatal ser uma condição usualmente benigna e classificada como icterícia fisiológica, ela também pode estar associada a fatores de risco e/ou patológicos e alcançar concentrações elevadas, atravessando a barreira hemato-encefálica, impregnando os núcleos da base kernicterus ou encefalopatia bilirrubínica aguda com sequelas neurológicas ${ }^{(3)}$.

A fototerapia é utilizada desde a década de 60 no tratamento da hiperbilirrubinemia e é a intervenção terapêutica de uso mais frequente no período neonatal( ${ }^{(4)}$. Alguns centros utilizam fototerapia em mais de $50 \%$ dos recém-nascidos prematuros ${ }^{(5)}$. Com o aprimoramento das técnicas e dos aparelhos, este procedimento tem apresentado maior eficácia com consequente redução no número de indicações de exsanguineotransfusão $0^{(3,6)}$.

Trata-se de uma técnica terapêutica cuja ação tem como princípio básico a transformação fotoquímica da estrutura da molécula da bilirrubina em produtos hidrossolúveis passíveis de eliminação renal e hepática. Somente a bilirrubina que está próxima à superfície da pele será alterada diretamente pela luz ${ }^{(7)}$.

Em 2004, a Academia Americana de Pediatria (AAP) publicou um guia de recomendações para o manejo do tratamento da hiperbilirrubinemia, adotado pela Sociedade Brasileira de Pediatria, em que considera o comprimento de onda ideal para absorção da bilirrubina entre de 425 a 475 nanômetros (nm), ou seja, na faixa azul ${ }^{(8)}$.

Para o sucesso da fototerapia, a assistência prestada pela equipe de Enfermagem deve levar em consideração alguns aspectos como: a superfície corporal do recém-nascido (RN) exposta à luz; a distância apropriada entre o $\mathrm{RN}$ e a fonte luminosa; a irradiância do aparelho de fototerapia; bem como cuidados com o paciente, desde a orientação sobre o contato dos pais com o $\mathrm{RN}$ e a proteção ocular do recém-nascido, até o controle dos sinais vitais e avaliação do balanço hídrico(1).

Uma pesquisa realizada na cidade do Rio de Janeiro, com o propósito de avaliação da prática clínica sobre o uso da fototerapia, na qual foram entrevistados 89 profissionais de saúde de 17 maternidades, constatou que vários aspectos deste procedimento são negligenciados. Embora os participantes afirmassem conhecer sobre a rotina escrita acerca do uso da fototerapia, observou-se o uso indevido de fraldas durante o procedimento, discordância no direcionamento ideal dos focos luminosos e discrepância das respostas fornecidas sobre a distância do RN e a lâmpada do aparelho de fototerapia. Os pesquisadores sugerem que todos os profissionais de saúde que manejam a fototerapia precisam ser informados das normas e rotinas existentes, conscientizados da necessidade de adesão às mesmas, treinados adequadamente para aplicá-las, além de avaliar regularmente a eficácia dessa modalidade terapêutica ${ }^{(9)}$.
Considerando que a fototerapia é a modalidade terapêutica mais utilizada para o tratamento da icterícia neonatal, ao mesmo tempo em que se observa a frequente desinformação acerca do seu uso ${ }^{(9)}$, justifica-se um estudo para investigar o conhecimento, a atitude e a prática dos profissionais de Enfermagem sobre a fototerapia. Por este motivo, este artigo tem como objetivo avaliar o conhecimento, atitude e prática sobre fototerapia entre profissionais de Enfermagem de dois hospitais de ensino da cidade de Recife.

Nessa perspectiva, este estudo pretende contribuir para a identificação de possíveis limitações no processo de trabalho, favorecendo o adequado manejo do recém-nascido portador de icterícia neonatal em tratamento com fototerapia, possibilitando a correção das fragilidades e limitações identificadas por meio da elaboração de treinamentos e da sistematização da assistência de Enfermagem ao recém-nascido em fototerapia.

\section{MÉTODOS}

Nesta pesquisa, foi realizado um estudo transversal do tipo inquérito CAP - Conhecimento, Atitude e Prática, sobre fototerapia entre profissionais de Enfermagem de Unidade Neonatal de dois hospitais de ensino: o Instituto de Medicina Integral Prof. Fernando Figueira (IMIP) e o Centro Integrado de Saúde Amaury de Medeiros (CISAM), localizados na cidade do Recife-PE.

O IMIP é uma instituição de direito privado sem fins lucrativos, enquanto o CISAM é uma instituição pública, pertencente à rede estadual de saúde, que presta assistência à população usuária do SUS e aos Servidores Estaduais do Estado de Pernambuco. A escolha dessas unidades hospitalares deveu-se ao fato de ambas serem hospitais de ensino, referências no estado de Pernambuco quanto à assistência à gestante de alto risco, bem como na capacitação de profissionais na área de Saúde da Mulher e da Criança, incluindo o Programa de Residência de Enfermagem, somados aos números de atendimentos a partos e assistência ao recém-nascido de alto risco com unidade neonatal interna.

A população do estudo foi censitária e constituída por 151 profissionais de Enfermagem (enfermeiros, auxiliares e técnicos de Enfermagem). Destes, um técnico de Enfermagem que atuava nas duas unidades foi entrevistado uma única vez; foram excluídos 31 profissionais, um deles por realizar consulta durante o momento em que respondia o questionário e os demais porque não estavam presentes no período da coleta dos dados (por motivo de férias, licença médica, licença maternidade e/ou licença prêmio). Além disso, houve oito recusas, sendo avaliados finalmente 111 profissionais.

Foram adotadas as seguintes definições para conhecimento, atitude e prática ${ }^{(10-12)}$ : conhecimento (capacidade de adquirir e reter informações a serem utilizadas, uma mistura de compreensão, experiência, discernimento e habilidade), atitude (inclinação para reagir de certo modo a certas situações; ver e interpretar eventos de acordo com certas predisposições; organizar opiniões dentro de uma estrutura inter-relacionada e coerente) e prática (aplicação de regras e conhecimentos que levam à execução da ação e maneira ética). 
Os dados foram coletados no período de dezembro de 2009 a março de 2010, por meio de questionário cuja elaboração aconteceu em três etapas. Na primeira etapa, foram observadas as recomendações para o uso e cuidados com a fototerapia de acordo com os manuais dos fabricantes dos aparelhos de fototerapia ${ }^{(13-15)}$. Em seguida, foi elaborado um formulário com perguntas relacionadas à atuação da Enfermagem na utilização dessa terapêutica, baseando-se no conteúdo programático da disciplina Saúde da Criança do curso de graduação e de nível médio em Enfermagem, com abordagem da temática "icterícia neonatal e fototerapia".

Em uma segunda etapa, o formulário foi submetido à validação de aparência e conteúdo realizado por enfermeiros com expertise teórico-prática na área de Pediatria de três instituições de Ensino Superior em Enfermagem do Recife. As perguntas foram pontuadas de acordo com o grau de importância para a construção do formulário ( 0 = Desnecessário, 1 = Regular, 2 = Bom, 3 = Ótimo), de forma a permanecerem aquelas que obtiveram pontuação máxima após duas rodadas de avaliação do instrumento pelos enfermeiros com expertise teórico-prática na área de Pediatria.

$\mathrm{Na}$ terceira etapa, o instrumento foi testado previamente com 20 profissionais de Enfermagem que não participaram do estudo, para avaliação quanto à objetividade, clareza das questões e tempo de preenchimento do questionário.

O questionário apresentou perguntas sobre características dos profissionais (idade, sexo e grau de especialização), o conhecimento e a atitude, com enfoque no manuseio ao recém-nascido em tratamento fototerápico e o uso da fototerapia no recém-nascido. Para as questões sobre conhecimento, foram atribuídas as respostas: "verdadeiro", "falso" e "não sei". Quanto à seção de atitude, utilizou-se a escala Likert $^{(16)}$ de cinco níveis ("concordo", "concordo plenamente, "discordo", "discordo plenamente" e "não tenho opinião"). Sobre a prática, as respostas foram do tipo dicotômica ("sim"/“não").

Após contato pessoal da pesquisadora com a equipe de Enfermagem de cada hospital no início de cada turno de plantão, os formulários foram distribuídos aos profissionais que concordaram participar do estudo mediante a assinatura do Termo de Consentimento Livre e Esclarecido, com a devida orientação do preenchimento no próprio local do trabalho, sem que fosse permitida a consulta em livros ou outras fontes de informação.

Para análise dos dados, considerou-se para o conhecimento: questão adequada quando a resposta foi "verdadeiro" para as assertivas verdadeiras ou "falso" para as assertivas falsas; questão não adequada quando a resposta foi "falso" ou "não sei" para as assertivas verdadeiras ou "verdadeiro" ou "não sei" para as assertivas falsas. Em relação à atitude: questão adequada quando a resposta foi "concordo" ou "concordo plenamente" para as assertivas verdadeiras ou "discordo" ou "discordo plenamente" para as assertivas falsas; questão não adequada quando a resposta foi "discordo", "discordo plenamente", "não tenho opinião" para as assertivas verdadeiras ou "concordo", "concordo plenamente", "não tenho opinião" para as assertivas falsas. Quanto à prática: questão adequada quando a resposta foi "sim" e questão inadequada quando a resposta foi "não". As respostas foram consideradas adequadas ou não adequadas de acordo com a literatura sobre assistência ao recém-nascido em fototerapia.

Para cada questionário foi calculada uma nota em uma escala de 0 a 10, sendo considerada a nota máxima 10 quando todas as questões apresentassem respostas corretas, definindo-se como ponto de corte um mínimo de $50 \%$ de acertos (média 5,0).

Foi criado um banco de dados para dupla digitação no Programa Epilnfo 3.4.3 e realizado teste $t$ de Student para comparação das médias de acertos, considerando nível de significância quando $p<0,05$. Os dados foram analisados e apresentados considerando dois grupos de profissionais: nível superior (enfermeiro) e nível médio (auxiliar e técnico de Enfermagem).

O projeto de pesquisa foi aprovado pelo Comitê de Ética em Pesquisa (CEP) do Instituto de Medicina Integral Prof. Fernando Figueira - IMIP, protocolo No 1327/2008.

\section{RESULTADOS}

Participaram do estudo 111 profissionais de Enfermagem, sendo $22(19,8 \%)$ profissionais do nível superior e $89(80,2 \%)$ de nível médio. Quanto ao local de trabalho, 72 (65\%) atuavam no IMIP e 39 (35\%) no CISAM.

A maioria dos profissionais de nível superior era do sexo feminino $(95 \%)$, com idade superior a 30 anos $(68,18 \%)$ e com mais de cinco anos de conclusão da graduação $(81,8 \%)$. Cerca de $20 \%$ mencionaram algum curso de especialização e $68,2 \%$ referiram ter acesso à informação sobre fototerapia durante a graduação.

Entre os profissionais de nível médio, observou-se novamente o predomínio do sexo feminino (95\%) e idade acima de 30 anos (56,1\%), com tempo de formação profissional superior a 5 anos (94,3\%); cerca de 8,9\% referiram algum curso de especialização. O acesso à informação sobre fototerapia na formação profissional foi referido por cerca de $80 \%$ desses profissionais.

Na tabela 1, observa-se a proporção de respostas adequadas às perguntas ou afirmações ao conhecimento sobre fototerapia, segundo o grau de formação profissional. O menor percentual de respostas adequadas foi observado quando se tratou do conhecimento sobre nível de irradiância mínima para troca de lâmpadas dos aparelhos e sobre a posição dos aparelhos em relação ao RN, tanto em relação aos profissionais de nível superior quanto ao nível médio.

$\mathrm{Na}$ tabela 2, tem-se a proporção das respostas adequadas às perguntas ou afirmações sobre atitude segundo os profissionais de nível superior e médio. Entre os profissionais de nível superior, das 11 assertivas, 7 apresentaram percentuais de acertos de $50 \%$ ou mais. Entre os profissionais de nível médio, das 11 , apenas 4 atingiram mais de $50 \%$.

No que diz respeito às assertivas: "Todo RN em fototerapia deve estar com a genitália coberta", "É importante o exame físico realizado pela Enfermagem ao $\mathrm{RN}$ exposto à fototerapia duas vezes ao dia" e "A higiene ocular do RN em fototerapia deve ser realizada com SF a 0,9\%", observamos nas duas 
Tabela 1 - Distribuição de respostas adequadas sobre o conhecimento em fototerapia entre profissionais de Enfermagem de dois hospitais de ensino. Recife-PE, 2009.

\begin{tabular}{|c|c|c|c|c|}
\hline \multirow{3}{*}{ Aspectos } & \multicolumn{2}{|c|}{ Nível Superior } & \multicolumn{2}{|c|}{ Nível Médio } \\
\hline & \multicolumn{2}{|c|}{ Respostas adequadas } & \multicolumn{2}{|c|}{ Respostas adequadas } \\
\hline & $N=22$ & $\%$ & $N=89$ & $\%$ \\
\hline \multicolumn{5}{|l|}{ Fatores que alteram a eficácia da fototerapia } \\
\hline Irradiância da fonte de luz & 22 & 100 & 56 & 62,9 \\
\hline Espectro da luz & 22 & 100 & 35 & 39,3 \\
\hline Tempo de uso das lâmpadas & 22 & 100 & 49 & 55,5 \\
\hline Foco luminoso distante do RN & 21 & 95,5 & 70 & 78,6 \\
\hline Área corporal exposta & 19 & 86,4 & 45 & 50,5 \\
\hline Concentração inicial da Bilirrubina & 8 & 36,4 & 10 & 11,2 \\
\hline Retirar RN da luz por tempo prolongado & 20 & 90,9 & 66 & 74,1 \\
\hline \multicolumn{5}{|c|}{ Distância do foco luminoso para o RN em centímetros } \\
\hline Fototerapia Convencional & 5 & 22,7 & 53 & 59,5 \\
\hline Fototerapia Bilispot $^{\oplus}$ & 2 & 12,5 & 13 & 14,6 \\
\hline Fototerapia Bilitron $^{\infty}$ & 5 & 12,5 & 6 & 6,7 \\
\hline \multicolumn{5}{|l|}{ Vestimenta do RN em uso da fototerapia } \\
\hline Totalmente despido & 4 & 18,1 & 12 & 13,5 \\
\hline Com fralda & 4 & 18,1 & 12 & 13,5 \\
\hline Com lençol & 22 & 100 & 87 & 97,7 \\
\hline \multicolumn{5}{|l|}{ Irradiância mínima para a troca de lâmpadas } \\
\hline Fototerapia Convencional & 1 & 4,5 & 1 & 1,12 \\
\hline Fototerapia Bilispot $^{\oplus}$ & 0 & - & 1 & 1,12 \\
\hline Fototerapia Bilitron $^{\infty}$ & 0 & - & 0 & - \\
\hline \multicolumn{5}{|l|}{ Mecanismo de ação da fototerapia } \\
\hline Fotoisomeriração e/ou fotooxidação & 16 & 72,2 & 19 & 21,3 \\
\hline \multicolumn{5}{|l|}{ Eventos adversos associados à fototerapia } \\
\hline Fezes diarréicas & 13 & 59,0 & 35 & 39,3 \\
\hline Lesão de retina & 19 & 86,4 & 67 & 75,2 \\
\hline Erupção cutânea & 13 & 59,0 & 36 & 40,4 \\
\hline Desidratação & 19 & 59,0 & 53 & 59,5 \\
\hline Comprometimento das gônadas & 12 & 54,5 & 52 & 58,4 \\
\hline \multicolumn{5}{|l|}{ Posição da fototerapia em relação ao $\mathrm{RN}$} \\
\hline Fototerapia convencional & 1 & 4,5 & 1 & 1,12 \\
\hline Fototerapia Bilispot & 1 & 4,5 & 2 & 2,24 \\
\hline Fototerapia Bilitron $^{\infty}$ & 1 & 4,5 & 1 & 1,12 \\
\hline
\end{tabular}

Fonte: Dados da pesquisa.

categorias profissionais baixos percentuais de respostas adequadas. Destaca-se ainda o percentual de apenas $5,6 \%$ para o grupo de nível médio de respostas adequadas no que se refere à higiene ocular com água fervida e $22,7 \%$ no que diz respeito à lesão de pele para o grupo de nível superior (Tabela 2).

Na tabela 3, estão apresentadas as proporções das respostas adequadas às perguntas ou afirmações sobre a prática, segundo os profissionais de nível superior e médio. No que se refere aos itens: "Anota no prontuário ao instalar o aparelho de fototerapia as condições da pele do RN" e "Verifica a temperatura do RN antes de instalar a fototerapia", observamos percentuais abaixo de $50 \%$ de respostas adequadas nas duas categorias profissionais.

Na tabela 4, estão apresentadas as médias das notas de conhecimento, atitude e prática dos profissionais de Enfermagem de acordo com a categoria profissional. No que se refere ao conhecimento, as notas foram maiores entre os profissionais de nível superior com tempo de graduação menor ou igual a 10 anos, com pós-graduação e com treinamento prévio em fototerapia. Observa-se diferença estatisticamente significante no que se refere a ter ou não pós-graduação $(p=0,017)$. Entre os profissionais de nível médio, observa-se resultado inverso quanto ao tempo de formado e possuir especialização técnica.

Para atitude e a prática, verificam-se maiores notas para os profissionais de nível superior com tempo de graduação acima de 10 anos, com pós-graduação e com treinamento prévio sobre fototerapia. Esse mesmo padrão é observado entre os profissionais de nível médio com exceção para a variável "pós- graduação" na avaliação da atitude.

Na tabela 5, observa-se a comparação das médias de conhecimento e prática. Para todas as variáveis, as diferenças de médias se mostraram estatisticamente significantes. 
Tabela 2 - Distribuição de respostas adequadas sobre a atitude em relação à fototerapia entre profissionais de Enfermagem de dois hospitais de ensino. Recife-PE, 2009.

\begin{tabular}{|c|c|c|c|c|}
\hline \multirow{3}{*}{ Assertivas } & \multicolumn{2}{|c|}{ Nível Superior } & \multicolumn{2}{|c|}{ Nível Médio } \\
\hline & \multicolumn{2}{|c|}{ Respostas adequadas } & \multicolumn{2}{|c|}{ Respostas adequadas } \\
\hline & $N=22$ & $\%$ & $N=89$ & $\%$ \\
\hline Considero importante a instalação da fototerapia pela enfermeira & 18 & 81,8 & 72 & 80,8 \\
\hline Acho que a fototerapia pode ser instalada por qualquer profissional de Enfermagem & 17 & 77,3 & 76 & 85,3 \\
\hline Todo RN em fototerapia deve estar com a genitália coberta & 5 & 22,7 & 12 & 13,4 \\
\hline $\begin{array}{l}\text { RN em fototerapia deve estar com máscara-ocular; se não houver } \\
\text { no serviço eu não instalo }\end{array}$ & 13 & 59,0 & 34 & 38,2 \\
\hline $\begin{array}{l}\text { RN em fototerapia eu considero como correto realizar mudança } \\
\text { de decúbito de } 4 \text { em } 4 \text { horas }\end{array}$ & 14 & 63,6 & 20 & 22,7 \\
\hline É comum lesão de pele no RN por queimadura de fototerapia & 5 & 22,7 & 60 & 67,4 \\
\hline $\begin{array}{l}\text { É importante o exame físico realizado pela Enfermagem ao RN } \\
\text { exposto a fototerapia duas vezes ao dia }\end{array}$ & 3 & 13,6 & 11 & 12,3 \\
\hline A falta da proteção ocular no RN em fototerapia causa lesão de retina & 22 & 100 & 84 & 94,4 \\
\hline $\begin{array}{l}\text { A higiene ocular do RN em fototerapia deve ser realizada } \\
\text { com água fervida na temperatura ambiente }\end{array}$ & 16 & 72,7 & 5 & 5,6 \\
\hline A higiene ocular do RN em fototerapia deve ser realizada com SF a 0,9\% & 5 & 22,7 & 18 & 20,2 \\
\hline Todo RN ictérico faz uso de fototerapia & 18 & 81,8 & 35 & 39,3 \\
\hline
\end{tabular}

Tabela 3 - Distribuição de respostas adequadas sobre a prática em fototerapia entre profissionais de Enfermagem de dois hospitais de ensino. Recife-PE, 2009.

\begin{tabular}{|c|c|c|c|c|}
\hline \multirow{3}{*}{ Assertivas } & \multicolumn{2}{|c|}{ Nível Superior } & \multicolumn{2}{|c|}{ Nível Médio } \\
\hline & \multicolumn{2}{|c|}{ Respostas adequadas } & \multicolumn{2}{|c|}{ Respostas adequadas } \\
\hline & $N=22$ & $\%$ & $N=89$ & $\%$ \\
\hline $\begin{array}{l}\text { Antes de instalar o aparelho de fototerapia, comunica aos pais } \\
\text { a respeito do procedimento }\end{array}$ & 14 & 63,6 & 62 & 69,6 \\
\hline Testa o aparelho de fototerapia antes de instalar no RN & 18 & 81,8 & 80 & 89,8 \\
\hline Antes de instalar o aparelho de fototerapia no RN, verifica a irradiância & 14 & 63,6 & 69 & 77,5 \\
\hline Antes de instalar o aparelho de fototerapia, certifica-se de que se encontra prescrito & 22 & 100 & 89 & 100 \\
\hline $\begin{array}{l}\text { Antes de instalar o aparelho de fototerapia, observa na prescrição } \\
\text { se o RN encontra-se em uso de pomadas, óleos, hidratantes e outros }\end{array}$ & 19 & 86,4 & 68 & 76,4 \\
\hline Anotar no prontuário o tipo de fototerapia e hora instalada & 12 & 54,5 & 65 & 73,0 \\
\hline $\begin{array}{l}\text { Anotar no prontuário, ao instalar o aparelho de fototerapia, } \\
\text { as condições da pele do RN }\end{array}$ & 8 & 36,4 & 22 & 24,7 \\
\hline Verifica a temperatura do $\mathrm{RN}$ antes de instalar a fototerapia & 7 & 31,8 & 33 & 37,0 \\
\hline $\begin{array}{l}\text { Concorda que é importante a realização do balanço hídrico para o } \\
\text { RN em uso de fototerapia }\end{array}$ & 21 & 95,5 & 72 & 80,8 \\
\hline
\end{tabular}


Tabela 4 - Distribuição das médias de conhecimento, atitude e prática sobre fototerapia segundo características de formação de profissionais de Enfermagem de dois hospitais de ensino. Recife-PE, 2009.

\begin{tabular}{|c|c|c|c|c|c|c|c|c|c|}
\hline \multirow{2}{*}{ Características } & \multicolumn{3}{|c|}{ Conhecimento } & \multicolumn{3}{|c|}{ Atitude } & \multicolumn{3}{|c|}{ Prática } \\
\hline & Nota & (DP) & $p$ & Nota & (DP) & $\boldsymbol{p}$ & Nota & (DP) & $p$ \\
\hline \multicolumn{10}{|c|}{ Nível de Instrução Superior } \\
\hline \multicolumn{10}{|c|}{ Tempo de graduação } \\
\hline$\leq 10$ anos & 3,9 & $(1,8)$ & 0,665 & 5,6 & $(2,0)$ & 0,98 & 6,0 & $(1,2)$ & 0,0059 \\
\hline$>10$ anos & 3,5 & $(1,4)$ & & 5,7 & $(1,4)$ & & 7,8 & $(1,5)$ & \\
\hline \multicolumn{10}{|l|}{ Pós-graduação } \\
\hline Sim & 4,0 & $(1,2)$ & 0,017 & 5,7 & $(2,0)$ & 0,8 & 7,0 & $(1,7)$ & 0,256 \\
\hline Não & 2,0 & $(2,0)$ & & 5,5 & $(1,0)$ & & 6,0 & $(1,1)$ & \\
\hline \multicolumn{10}{|c|}{ Treinamento prévio sobre fototerapia } \\
\hline Sim & 4,4 & $(1,0)$ & 0,517 & 6,7 & $(1,2)$ & 0,3 & 7,4 & $(1,8)$ & 0,63 \\
\hline Não & 3,7 & $(1,6)$ & & 5,5 & $(1,8)$ & & 6,8 & $(1,7)$ & \\
\hline \multicolumn{10}{|c|}{ Nível de Instrução Médio } \\
\hline \multicolumn{10}{|c|}{ Tempo de formado } \\
\hline$\leq 10$ anos & 2,3 & $(1,0)$ & 0,93 & 4,8 & $(1,3)$ & 0,08 & 6,6 & $(2,0)$ & 0,84 \\
\hline$>10$ anos & 2,3 & $(1,4)$ & & 5,3 & $(1,6)$ & & 6,7 & $(1,6)$ & \\
\hline \multicolumn{10}{|c|}{ Especialização técnica } \\
\hline Sim & 1,8 & $(0,8)$ & 0,236 & 4,2 & $(1,0)$ & 0,16 & 7,3 & $(2,2)$ & 0,356 \\
\hline Não & 2,4 & $(1,2)$ & & 5,0 & $(1,4)$ & & 6,6 & $(1,9)$ & \\
\hline \multicolumn{10}{|c|}{ Treinamento sobre fototerapia } \\
\hline Sim & 2,4 & $(1,5)$ & 0,636 & 5,0 & $(1,2)$ & 0,88 & 6,8 & $(1,8)$ & 0,66 \\
\hline Não & 2,2 & $(1,2)$ & & 4,8 & $(1,4)$ & & 6,5 & $(1,9)$ & \\
\hline
\end{tabular}

Fonte: Dados da pesquisa.

Tabela 5 - Comparação das médias de conhecimento e prática sobre fototerapia segundo características de formação de profissionais de Enfermagem de dois hospitais de ensino. Recife-PE, 2009

\begin{tabular}{|c|c|c|c|c|c|c|c|}
\hline \multirow{2}{*}{ Características } & \multicolumn{2}{|c|}{ Conhecimento } & \multicolumn{2}{|c|}{ Prática } & \multicolumn{2}{|c|}{ Diferença } & \multirow{2}{*}{$\begin{array}{c}\text { Valor de } p \\
\text { (2) }\end{array}$} \\
\hline & Média & (DP) & Média & (DP) & Média & (DP) (1) & \\
\hline \multicolumn{8}{|c|}{ Nível de Instrução Superior } \\
\hline \multicolumn{8}{|c|}{ Tempo de graduação } \\
\hline$\leq 10$ anos & 3,9 & $(1,8)$ & 6,0 & $(1,2)$ & 2,1 & $(2,0)$ & $<0,0058$ \\
\hline$>10$ anos & 3,6 & $(1,4)$ & 7,8 & $(1,6)$ & 4,3 & $(1,5)$ & $<0,001$ \\
\hline \multicolumn{8}{|l|}{ Pós-graduação } \\
\hline Sim & 4,0 & $(1,0)$ & 7,4 & $(1,8)$ & 3,2 & $(2,0)$ & $<0,001$ \\
\hline Não & 2,0 & $(2,0)$ & 6,0 & $(1,0)$ & 4,0 & $(1,0)$ & $<0,0037$ \\
\hline \multicolumn{8}{|c|}{ Treinamento prévio sobre fototerapia } \\
\hline Sim & 4,0 & $(1,3)$ & 7,0 & $(1,8)$ & 3,0 & $(1,8)$ & $<0,001$ \\
\hline Não & 3,7 & $(1,7)$ & 7,0 & $(1,5)$ & 3,3 & $(2,7)$ & $<0,0347$ \\
\hline \multicolumn{8}{|c|}{ Nível de Instrução Médio } \\
\hline \multicolumn{8}{|c|}{ Tempo de graduação } \\
\hline$\leq 10$ anos & 2,3 & $(1,1)$ & 6,6 & $(2,0)$ & 4,3 & $(1,9)$ & $<0,001$ \\
\hline$>10$ anos & 2,3 & $(1,4)$ & 6,7 & $(1,6)$ & 4,4 & $(1,9)$ & $<0,001$ \\
\hline \multicolumn{8}{|c|}{ Especialização técnica } \\
\hline Sim & 3,0 & $(1,3)$ & 7,3 & $(1,7)$ & 4,3 & $(1,8)$ & $<0,001$ \\
\hline Não & 1,8 & $(0,8)$ & 7,2 & $(2,0)$ & 5,4 & $(1,7)$ & $<0,001$ \\
\hline \multicolumn{8}{|c|}{ Treinamento sobre fototerapia } \\
\hline Sim & 2,2 & $(1,1)$ & 6,6 & $(1,9)$ & 4,4 & $(2,0)$ & $<0,001$ \\
\hline Não & 2,5 & $(1,6)$ & 6,6 & $(1,5)$ & 4,1 & $(1,6)$ & $<0,001$ \\
\hline
\end{tabular}

Fonte: Dados da pesquisa.

(1) Prática - Conhecimento

(2) Teste $t$ de Student 


\section{DISCUSSÃO}

De acordo com os resultados desse estudo, o achado mais relevante está na constatação de que, apesar dos profissionais em geral, tanto do grupo do nível superior quanto do nível médio, terem demonstrado uma média abaixo de 5,0 na avaliação do conhecimento, essa média foi diferente em todas as categorias, quando comparada a sua prática sobre o uso da fototerapia (mínimo de 6,0 e máximo de 7,4). Esses achados podem estar relacionados ao fato de que as capacitações em serviço oferecidas pelas unidades hospitalares evidenciam muito mais as questões práticas, oferecendo pouca oportunidade para os profissionais de Enfermagem aprimorarem seus conhecimentos teóricos, podendo ocorrer, assim, o distanciamento dos profissionais de Enfermagem do conhecimento científico e uma maior aproximação com a prática.

Ressalta-se também que, quanto à atitude, as médias para o grupo de nível superior foram todas acima de 5,0, enquanto no grupo de nível médio, mesmo não alcançando todas as médias de 5,0 ou mais, a mínima foi de 4,2, diferente das médias do conhecimento que variaram de 2,4 (a mínima) à 1,8 (a máxima). Por outro lado, em estudo realizado com a metodologia CAP(17), verificou-se conhecimento adequado, prática não eficiente e atitude com necessidade de mudança, o que é encontrado na maioria dos estudos CAP. Talvez os achados do estudo atual reflitam a opção de formatação das questões utilizadas para essa avaliação, cujas alternativas de respostas para o conhecimento foram mais explicativas, menos sujeitas a respostas ao acaso.

No grupo estudado, observou-se a predominância do sexo feminino, o que pode estar relacionado à questão de gênero na profissão da Enfermagem, ainda nos tempos atuais em que observamos uma escassa participação dos homens nas unidades neonatais ${ }^{(18)}$.

Houve um baixo percentual de especialização entre os profissionais de nível superior assim como de cursos de especialização técnica para o nível médio. Especula-se que esse fato possa dever-se a falta de cursos de especialização específicos em neonatologia no estado de Pernambuco.

Apesar da eficácia da fototerapia ser avaliada pelo comprimento da luz, irradiância espectral e superfície corpórea exposta a luz ${ }^{(2,19)}$, identificou-se que alguns profissionais do nível médio não citaram a irradiância e o espectro da luz como fatores que interferem na eficácia desta terapêutica, assim como outros aspectos, tais como, foco luminoso e a distância para o recém-nascido e a área corporal exposta.

Em relação à distância do foco luminoso para o $\mathrm{RN}$ e irradiância mínima para troca das lâmpadas, chamamos atenção para o baixo percentual das respostas adequadas, nas duas categorias profissionais. Isso de deve possivelmente ao fato de existirem nos dois serviços (IMIP e CISAM) profissionais específicos que monitoram o posicionamento e a medição da irradiância dos aparelhos de fototerapia, lembrando que ao posicionar o recém-nascido a uma distância inadequada do foco luminoso recomendada, pode ocorrer o risco de queimadura no $\mathrm{RN}^{(1,6)}$.

Chamamos atenção para os achados em relação ao mecanismo de ação da fototerapia, uma vez que foi observado seu desconhecimento nas duas categorias profissionais (nível superior e médio). Nesse sentido, frisamos a grande importância para os profissionais de Enfermagem de conhecerem o mecanismo de ação de qualquer tratamento a ser administrado, podendo melhorar a eficiência da terapêutica usada.

Em relação aos eventos adversos associados à fototerapia, $54 \%$ dos participantes de nível superior e $58,4 \%$ dos participantes de nível médio responderam adequadamente sobre o risco de comprometimento das gônadas, apesar de alguns autores citarem não haver esse risco, já que o comprimento de onda utilizado na fototerapia penetra apenas 2 a $3 \mathrm{~mm}$ na pele ${ }^{(6)}$.

Em estudo realizado a fim de avaliar a prática clínica com o uso da fototerapia em recém-nascido, $30 \%$ dos entrevistados recomendaram o uso rotineiro de fraldas durante o uso da fototerapia ${ }^{(9)}$. Apesar de alguns achados relativos à prática ou à atitude serem provenientes da falta do conhecimento da terapêutica, um percentual elevado das duas categorias concorda que todo RN deve estar com a genitália coberta.

Os achados da presente análise demonstram baixo percentual de resposta adequada para o posicionamento do recém-nascido em tratamento com fototerapia: somente 4,5\% dos participantes de nível superior responderam adequadamente, $1,12 \%$ dos participantes de nível médio responderam para dois tipos de aparelhos e 2,24\% para um tipo. Aqui, é importante ressaltar que quanto maior a superfície exposta à luz, maior a eficácia da fototerapia ${ }^{(9,19)}$.

Ainda em relação ao posicionamento do recém-nascido, o manual do fabricante orienta que deve ser posicionado de forma que a radiação incida em todo o seu corpo, para que haja o aproveitamento do foco luminoso na fototerapia convencional. Já para o Bilispot $^{\circledR}$ e Bilitron ${ }^{\circledR}$ a luz deverá incidir desde o tórax até a raiz das $\operatorname{coxas}^{(13,15)}$.

Um aspecto que deve ser destacado é o relato da não instalação do aparelho de fototerapia quando há a falta do protetor ocular para o RN no serviço hospitalar. Nesse quesito, chamamos a atenção para o percentual de $59,6 \%$ dos profissionais de nível superior e $38,2 \%$ de nível médio que responderam adequadamente, sendo preferível improvisar um protetor ocular a deixar de instalar a fototerapia(8).

Sobre a prática, algumas medidas são de fundamental importância. Neste sentido, os resultados demonstram que somente $31,8 \%$ dos profissionais de nível superior e $37 \%$ do nível médio verificavam a temperatura do $\mathrm{RN}$ antes de instalar a fototerapia. A hipertermia é um dos efeitos colaterais mais comuns da fototerapia, já que alguns aparelhos de fototerapia provocam elevação da temperatura com consequente aumento do consumo de oxigênio, da frequência respiratória e do fluxo sanguíneo na pele, culminando em maior perda insensível de água ${ }^{(2-3)}$. Outro achado importante se relaciona à anotação no prontuário acerca das condições da pele do recém-nascido antes de instalar a fototerapia, quesito em que as duas categorias profissionais apresentaram um baixo percentual de respostas adequadas.

Ao se considerar o conhecimento, a atitude e a prática à luz de algumas características de formação dos profissionais como o tempo de graduação, a realização de curso de pós-graduação e de treinamento específico em fototerapia, verificou-se 
melhor desempenho relacionado à prática, seguindo-se da atitude e do conhecimento para ambos os grupos de profissionais (superior e médio). Em relação ao conhecimento, os profissionais de nível superior com tempo de graduação inferior a 10 anos, com pós-graduação e treinamento prévio em fototerapia obtiveram maiores notas. Já em relação à atitude e à prática, as notas foram maiores para aqueles com tempo de graduação acima de 10 anos.

É possível que um maior tempo de formação e, consequentemente, uma maior experiência prática tenha influenciado o melhor desempenho quanto à atitude e à prática. Esse aspecto é mais evidente entre os profissionais de nível médio: aqueles com tempo de formado acima de 10 anos obtiveram melhor desempenho, não apenas para atitude e prática, mas também em relação ao conhecimento.

Algumas limitações do estudo devem aqui ser pontuadas. Apesar dos critérios adotados para a elaboração dos formulários para coleta de dados, considerando o consenso realizado entre especialistas da área de Enfermagem, é possível que o formato das questões tenha influenciado nas respostas dos profissionais. Levando em consideração o fato de os profissionais estarem cientes de que o objetivo do estudo seria de avaliar o seu conhecimento, atitude e prática, é possível que tenham tendido a afirmar que realizam determinadas práticas, embora este aspecto tenha sido minimizado pela forma de coleta dos dados (formulário auto-preenchido anonimamente). Ademais, a avaliação da atitude e da prática apenas baseada nas informações dos profissionais sem a observação direta dos mesmos no manejo da fototerapia é uma limitação que deve também ser considerada.

Apesar dessas limitações, o estudo apresentou resultados que podem ser úteis para a organização dos serviços de saúde no que se refere à qualificação profissional, suscitando novas questões de pesquisa com outras abordagens metodológicas. As lacunas de conhecimento, atitude e prática observadas podem contribuir na identificação de pontos a serem desenvolvidos e enfatizados nos diversos programas de educação continuada para os profissionais de Enfermagem.

\section{REFERÊNCIAS}

1. Almeida MFB, Nader PJN, Draque CM. Icterícia neonatal. In: Campos Jr D, Lopez FA, editores. Tratado de Pediatria da Sociedade Brasileira de Pediatria. $2^{\mathrm{a}}$ ed. Barueri: Manole; 2010. p.1513-26.

2. Almeida MFB, Draque CM. Neonatal Jaundice and breastfeeding. NeoReviews 2007;8:282-8.

3. American Academy of Pediatrics. Subcommittee on hyperbilirubinemia. Management of hyperbilirrubinemia in the newborn infant 35 or more weeks of gestation. Pediatrics 2004;114:297-316.

4. Petrova A, Mehta R, Birchwood G, Ostfeld B, Hegyi T. Management of neonatal hyperbilirubinemia: Pediatricians' practices and educational needs. BMC pediatr 2006;6:1186-1471.

5. Dani C, Poggi C, Barp J, Romagnoli C, Buonocore G. Current Italian practices regarding the management of hyperbilirubinaemia in preterm infants. Acta Paediatr 2011;100(5):666-9.

6. Mishra S, Agarwal R, Deorari AK, Paul VK. Jaundice in the Newborns. Indian J Pediatr 2008;75(2):157-63.

7. Maisels MJ. Icterícia. In: Macdonald MG, Seshia, MMK, Mullett, MD. Fisiopatologia e tratamento do Recém-nascido. $6^{\text {a }}$ ed. Rio de Janeiro: Guanabara Koogan; 2007. p. 703-77.

8. Silva L, Silva FS, Turiani M, Juliani CMCM, Spiri WM. Desenvolvimento de um protetor ocular para fototerapia em recém-nascido: uma tecnologia. Rev Latinoam Enferm 2008;16(1):47-51.

9. Vieira AA, Lima CLMA, Carvalho M, Moreira MEL. O uso da fototerapia em recém-nascidos: avaliação da prática clinica. Rev Bras Saúde Matern Infant 2004;4(4):359-66.

10. Badran IG, Knowledge, attitude and practice the three pillars of excellence and wisdom: a place in the medical profession. East Mediterr Health J 1995; 1:S8-16.

11. Gonçalves VLM, Leite MMJ. Instrumento para mensuração de atitude frente ao processo de avaliação e desempenho. Rev Bras Enferm 2005;58(5):563-7.

12. Acioli S. A prática educativa como expressão do cuidado em saúde pública. Rev Bras Enferm 2008;61(1):117-21.

13. Manual do Usuário: Bilitron Modelos 3006 BTI, 3006 BTP e 3006 BTB Fanem (fototerapia azul), Bilispot Mo- $^{\circledast}$ delos 006 BB e 006 BP Fanem (fototerapia halógena). São Paulo; 2009.

14. Manual do Usuário: Fototerapia convencional modelos Gigante F6-P, F7-P. F8-P. São Paulo; 2000.

15. Manual do Usuário: Fototerapia convencional modelo Olideff 93 k 108. São Paulo; 2000.

16. Likert R. A Technique for the measurement of Attitudes. Arch Psychol 1932;140:1-50.

17. Lopes MHB de Moraes, Alves AS. Conhecimento, atitude e prática do uso de pílula e preservativo entre adolescentes universitários. Rev Bras Enferm 2008;61(2):46-51.

18. Coelho EAC, Gênero, saúde e Enfermagem. Rev Bras Enferm 2005;58(3):345-8.

19. Ferreira ALC, Nascimento RM, Veríssimo RCSS. Irradiance of phototherapy equipment in maternity wards in Maceió. Rev Latinoam Enferm 2009;17(5):695-700. 